\title{
亚甲基蓝/水溶性磷酸盐柱[5]芳烃对百草枯的竞争性苂光传感
}

\author{
杨云汉保秋连罗建萍杨俊丽李灿花魏可可 \\ 钏永明杨丽娟* \\ (云南民族大学化学与环境学院 云南省高校智能超分子化学重点实验室 \\ 生物基材料绿色制备技术国家地方联合工程中心＼cjkstart昆明 650500)
}

\begin{abstract}
摘要 利用水溶性磷酸盐柱 [5]芳烃(PP5A)作为主体，指示染料亚甲基蓝(MB)作为客体，构筑了 MB/PP5A 主-客体包合 物, 研究了 PP5A 与 MB 包合时的苂光性质、络合常数、络合比以及包合模式, 并将包合物运用于百草枯 $(\mathrm{PQ})$ 的竞争性 苂光传感. MB 与 PP5A 受体络合后, MB 的苂光发生淬灭, 将 PQ 加入到 MB/PP5A 传感系统中, PQ 竞争进入 PP5A 的 空腔中, 而 MB 从 PP5A 的空腔中脱离导致荧光恢复, 以此来实现对 PQ 的竞争性荧光传感. 因此, MB/PP5A 荧光传感 系统可以灵敏地检测 $P Q$, 最低检测限为 $3.6 \times 10^{-7} \mathrm{~mol} \cdot \mathrm{L}^{-1}$. 该探针具有选择性强、制备简便快捷、较宽 $\mathrm{pH}$ 范围下响 应和良好的抗干扰性能等优点, 为生物体及环境中 $P Q$ 的传感检测提供基础.
\end{abstract}

关键词 水溶性磷酸盐柱 [5]芳烃; 亚甲基蓝; 包合物; 竞争识别; 百草枯

\section{Competitive Fluorescence Sensing for Paraquat Based on Methylene Blue/Water-Soluble Phosphate Salt Pillar[5]arene}

\author{
Yang, Yunhan \\ Bao, Qiulian \\ Luo, Jianping \\ Yang, Junli \\ Li, Canhua \\ Wei, Keke \\ Chuan, Yongming \\ Yang, Lijuan*
}

(Key Laboratory of Intelligent Supramolecular Chemistry at the University of Yunnan Province, National and Local Joint Engineering Research Center for Green Preparation Technology of Biobased Materials, School of Chemistry \& Environment, Yunnan Minzu University, Kunming 650500)

\begin{abstract}
Using water-soluble phosphate salt pillar[5]arene (PP5A) as the host and indicate dye methylene blue (MB) as the guest, the MB/PP5A host-guest inclusion complex was constructed. The fluorescence properties, complexation constants, complexation ratios and binding modes of PP5A and MB were studied. The inclusion complex was applied to competitive fluorescent sensing of paraquat (PQ). When MB is complexed with PP5A receptor, its fluorescence is quenched. When PQ is added to the MB/PP5A sensing system, PQ competes into the cavity of PP5A and MB is detached from the cavity of PP5A, which led to fluorescence recovery. This enables competitive fluorescence sensing of PQ. Therefore, the MB/PP5A fluorescence sensing system can detect PQ sensitively with a minimum detection limit of $3.6 \times 10^{-7} \mathrm{~mol} \cdot \mathrm{L}^{-1}$. The probe has the advantages of strong selectivity, simple and fast preparation, response in a wide $\mathrm{pH}$ range and good anti-interference performance, and can provide the basis for the sensing detection of PQ in organisms and environments.

Keywords water-soluble phosphate salt pillar[5]arene; methylene blue; inclusion complex; competitive recognition; paraquat
\end{abstract}

百草枯(也称紫精，1,1-二甲基-4,4-二氯双吡啶， Paraquat，简称 PQ)，是一种非选择性、高效、速效的除 草剂 ${ }^{[1]}$. 自 1962 年以来, 被 130 多个国家的农业和非农 业地区广泛使用 ${ }^{[2]}$. 然而, PQ 的毒性大且口服中毒死亡
率高, 达 $90 \%{ }^{[3]}$. 由于自杀或意外接触 PQ 而导致的死亡 病例急剧增加, 我国已在 2016 年 7 月 1 日停止了 PQ 的 水剂在国内的销售和使用.目前，临床上还没有有效治 疗 PQ 中毒的解毒剂，但有研究表明部分大环化合物(如

\footnotetext{
* Corresponding author. E-mail: yangljyang@sina.com

Received November 6, 2019; revised December 18, 2019; published online March 6, 2020

Project supported by the National Natural Science Foundation of China (Nos. 21562048, 21762051), the Program for Innovative Research Team (in Science and Technology) in University of Yunnan Province and the Science Research Fund Project of Yunnan Provincial Department of Education (No. 2019Y0193). 国家自然科学基金(Nos. 21562048, 21762051)、云南省高校有机功能分子及材料科技创新团队和云南省教育厅科学研究基金(No. 2019Y0193)资助项 目.
} 


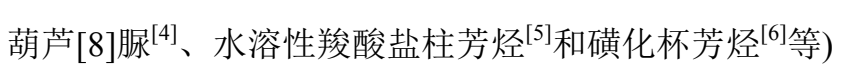
对 PQ 的毒性有较好的抑制作用. 由于 PQ 的高水溶性, 它对农业用水、饮用水和地下水都有潜在的污染威胁 ${ }^{[7]}$, 虽然残留的 PQ 可以通过光化学和微生物降解, 但是降 解过程十分缓慢 ${ }^{8]}$. 迄今为止, 研究人员已开发了许多 用于识别和测定 PQ 的分析方法, 如分光光度法 ${ }^{[9]}$ 、拉曼 光谱法 ${ }^{[10]}$ 、液相色谱法 ${ }^{[11]}$ 、气相色谱法 ${ }^{[12]}$ 、电化学法 ${ }^{[13]}$ 、 电泳法 ${ }^{[14]}$ 、电喷雾电离一质谱串联法 ${ }^{[15]}$ 等, 虽然这些检 测技术具有较高的选择性和灵敏度, 但是依然存在稳定 性差、所需仪器昂贵和耗时等不足. 因此, 开发一种灵 敏度高、选择性强、操作简便且快捷有效的检测方法十 分有意义.

大环化合物是一系列具有重复单元的环状低聚物, 四代经典的大环化合物包括冠醚、环糊精、杯芳烃以及 葫芦嘱 ${ }^{[16 ~ 19]}$. 由于其具有独特的主-客键合能力, 常被

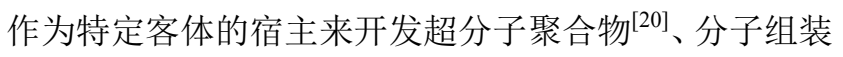

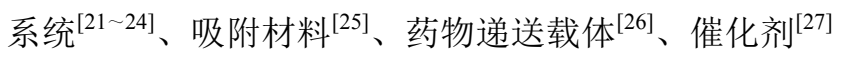
和分子识别体系 ${ }^{[28,29]}$. 近年来, 基于柱芳烃的 PQ 识别 探针已被广泛报道, Tang 和 Yang 等 ${ }^{[30,31]}$ 构筑了基于水溶 性磷酸盐柱 [5]和柱 [6]芳烃与石墨烯的纳米复合材料, 实现了对 $\mathrm{PQ}$ 的竞争性荧光检测及电化学传感. Yang 等 ${ }^{[32,33]}$ 制备了水溶性柱 [5]和柱[6]芳烃的功能化金纳米 离子, 用于对 PQ 的超灵敏检测. Sun 等 ${ }^{[34]}$ 通过葫芦 [8] 嘱与亚甲基蓝的包合物建立了一种 PQ 的竞争识别方法, 能有效识别活细胞中和老鼠体内的 PQ. Mao 等 ${ }^{[35]}$ 开发 了一种肼基柱 [5]芳烃功能化石墨烯的荧光传感材料, 该方法可应用于活细胞及老鼠体内 PQ 的检测. 2008 年, Ogoshi 等 ${ }^{[36]}$ 以廉价、简单的芳香族为原料, 制备出了新 顷芳香族大环化合物柱 $[n]$ 芳烃. 该大环的特征是具有 高度对称性的刚性富电子空腔结构, 由于空腔的两端均 具有负电性较强的氧原子, 即使不经修饰也能对阳离子 和中性客体小分子表现出优越的主客体性能 ${ }^{[37,38]}$. 经过 十多年的发展, 柱芳烃修饰的金属纳米离子 ${ }^{[39]}$ 、苂光基 团功能化柱芳烃 ${ }^{[40]}$ 、柱芳烃凝胶 ${ }^{[41]}$ 等已经在金属离子、 生物分子和农药的检测与识别中得到广泛应用.

本文首次构筑了水溶性磷酸盐柱 [5]芳烃(Watersoluble phosphate salt pillar[5]arene, 简称 PP5A)与亚甲 基蓝(Methylene blue, 简称 MB)的包合物 MB/PP5A, 深 入研究了 PP5A 与 MB 包合时的苂光性质、络合常数、 络合比以及包合模式, 并将其作为荧光探针用于 $P Q$ 的 竞争性苂光传感. 如 Scheme 1 所示, 指示染料 MB 首先 与 PP5A 受体络合, MB 进入 PP5A 的空腔后形成包合物, $M B$ 的苂光发生淬灭. 然后将 $\mathrm{PQ}$ 加入到 MB/PP5A 传感 系统中, PQ 竞争进入 PP5A 的空腔中, MB 从 PP5A 的空 腔中脱离导致苂光恢复. 该方法具有操作简单、选择性
强、成本低等优点, 为生物体及环境中 PQ 的传感检测 提供基础.

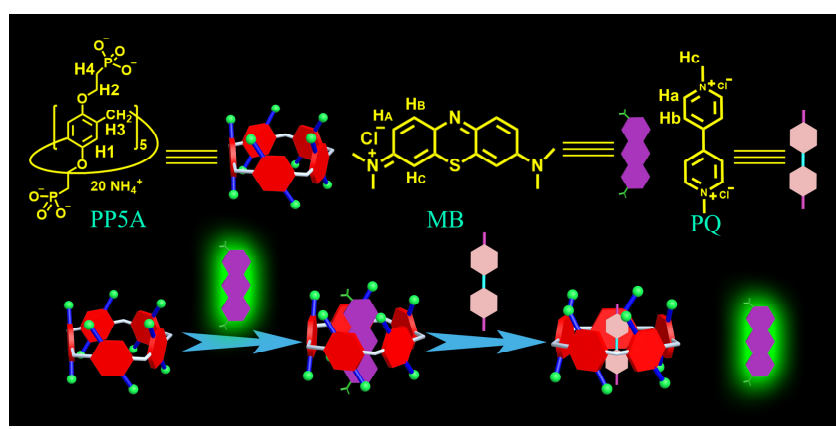

图式 1 PP5 $、$ 、 $\mathrm{MB} 、 \mathrm{PQ}$ 的结构以及 $\mathrm{MB} / \mathrm{PP} 5 \mathrm{~A}$ 对 $\mathrm{PQ}$ 的竞争 性荧光传感示意图

Scheme 1 Structures of PP5A, MB and PQ, and the schematic diagram of competitive fluorescence sensing of MB/PP5A for PQ

\section{1 结果与讨论}

\subsection{PP5A 与 MB 的主客体络合研究}

准确配制 $0.2 \mathrm{mmol} \cdot \mathrm{L}^{-1} \mathrm{MB}$ 和 $0.5 \mathrm{mmol} \cdot \mathrm{L}^{-1} \mathrm{PP} 5 \mathrm{~A}$ 的水溶液, 分别移取 $250 \mu \mathrm{L} \mathrm{MB}$ 和 $20 \sim 1000 \mu \mathrm{L}$ PP5A 于 $10 \mathrm{~mL}$ 容量瓶中, 超纯水定容、摇匀, 静置 $30 \mathrm{~min}$ 后 测定其苂光光谱. 苂光光谱仪的参数设置为: 激发波长 $625 \mathrm{~nm}$ 、激发狭缝和发射狭缝均为 $10 \mathrm{~nm}$. PP5A 与 MB 的苂光滴定曲线如图 1A 所示, 随着 PP5A 的浓度的增 加, $\mathrm{MB}$ 在 $684 \mathrm{~nm}$ 处的苂光强度逐渐减弱，说明阳离子 型染料 MB 与阴离子型大环 PP5A 之间存在较强的相互 作用.

通过等摩尔连续变化法 ${ }^{[42]}$ 进一步确定 MB 与 PP5A 的络合比. 准确配制 $50 \mathrm{~mL} 5 \times 10^{-6} \mathrm{~mol} \cdot \mathrm{L}^{-1}$ 的 $\mathrm{MB}$ 和 PP5A 溶液, 移取 $5.0 \sim 0 \mathrm{~mL} \mathrm{MB}$ 溶液于 $5 \mathrm{~mL}$ 比色管中, 然后分别移取 $0 \sim 5.0 \mathrm{~mL}$ 的 PP5A 溶液到相应的比色管 中, 从而保证溶液总体积为 $5.0 \mathrm{~mL}$, 总浓度为 $5 \times 10^{-6}$ $\mathrm{mol} \cdot \mathrm{L}^{-1}$. 混合摇匀静置 $30 \mathrm{~min}$ 后测定荧光光谱. 图 $1 \mathrm{~B}$ 为 MB/PP5A 体系的 Job's 曲线, 显然, 当比例系数为 0.5 时, 比例系数与苂光强度之差达到最大值, 由此可以推 断出 $\mathrm{MB}$ 与 $\mathrm{PP} 5 \mathrm{~A}$ 的络合比为 $1: 1$.

此外, 采用等温滴定量热法(ITC)测定水溶液中 PP5A 与 MB 的络合常数, 将主体 PP5A $\left(6 \mathrm{mmol} \cdot \mathrm{L}^{-1}\right)$ 溶 液逐渐加入到 $\mathrm{MB}$ 溶液 $\left(0.6 \mathrm{mmol} \cdot \mathrm{L}^{-1}\right)$ 中, 单次滴定体 积为 $1.5 \mu \mathrm{L}$ ，连续滴定 25 次，每次滴定时间间隔为 120 $\mathrm{s}$, 每次滴定 $3 \mathrm{~s}$ 完成. 量热滴定曲线如图 2 所示, PP5A 与 $\mathrm{MB}$ 的滴定数据根据“一组拟合位点”的模式拟合, 得 到 PP5A 与 $\mathrm{MB}$ 的络合常数为 $(1.98 \pm 0.501) \times 10^{4} \mathrm{~L}$. $\mathrm{mol}^{-1}$. 从 ITC 的滴定突跃点得知 PP $5 \mathrm{~A}$ 与 MB 的络合比 为 $1: 1$, 与等摩尔连续变化法的分析结果一致. 

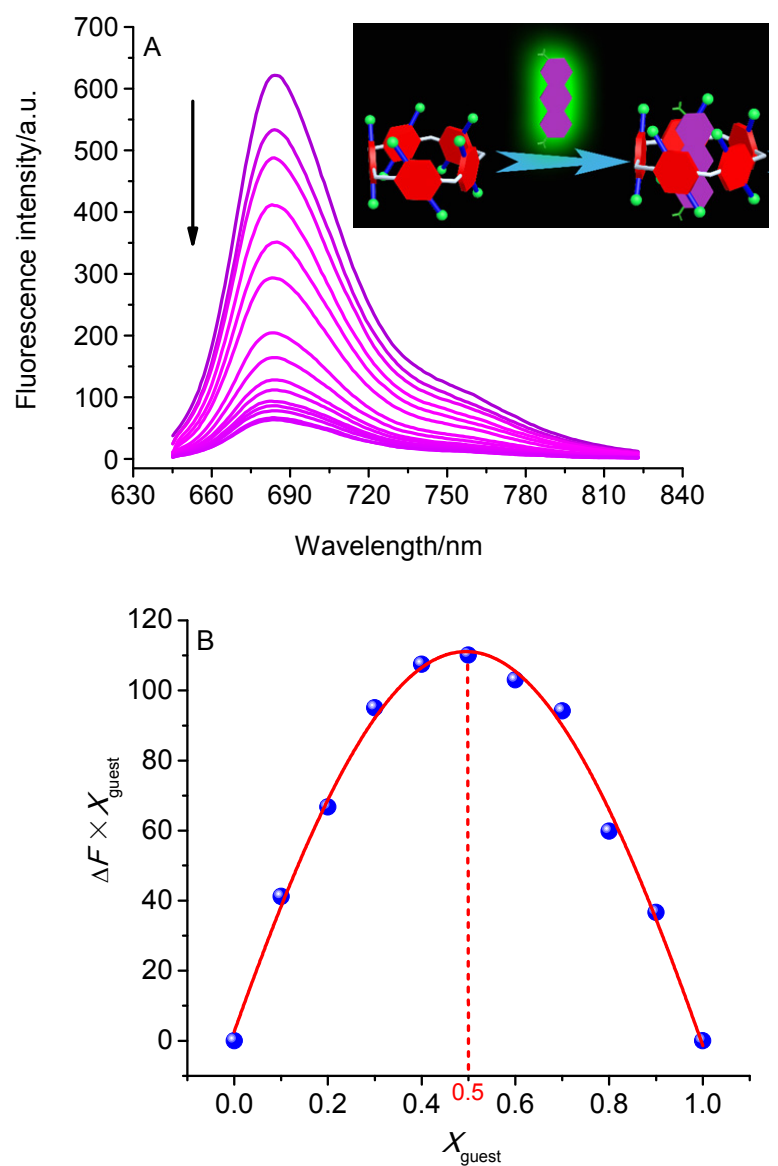

图 $1 \mathrm{MB}$ 与 PP5A 的苂光滴定图(A)和 Job's 曲线(B)

Figure 1 Fluorescence titration diagram of MB and PP5A (A) and Job's plot (B) of MB/PP5A inclusion complex

Huang 等 ${ }^{[43]}$ 采用 ITC 滴定得到 PP5A 与 PQ 的络合

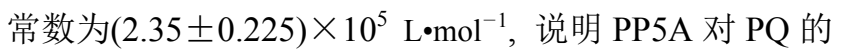
络合能力强于 MB. 通过分析 PP5A 与二者的 ITC 数据 可知: $\Delta H^{\mathrm{o}}<0, T \Delta S^{\mathrm{o}}<0,\left|\Delta H^{\mathrm{o}}\right|>\left|T \Delta S^{\mathrm{o}}\right|$, 说明 PP5A 与 $\mathrm{MB}$ 和 $\mathrm{PQ}$ 的络合主要为焓驱动过程. 此外, 为了对比不同 大环宿对 MB 的络合能力差异, 表 1 列举了不同大环主 体，如葫芦[7]艮(CB7)、葫芦 [8]腿(CB8)、磺化杯 [4]芳烃 $(\mathrm{SC} 4 \mathrm{~A}) 、$ 磺化杯 $[6]$ 芳烃 $(\mathrm{SC} 4 \mathrm{~A}) 、 \beta$-环糊精 $(\beta \mathrm{CD})$ 和水溶 性羧酸盐柱 [6]芳烃(WP6A)对 MB 的结合常数.

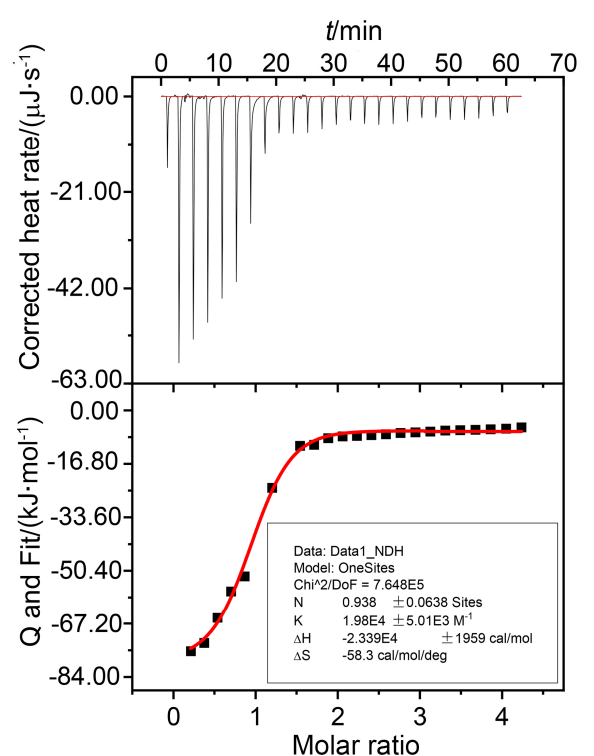

图 2 PP5A 与 $\mathrm{MB}(\mathrm{A})$ 和 $\mathrm{PQ}(\mathrm{B})$ 在水溶液中的微量热滴定 $(298.15 \mathrm{~K})$

Figure 2 Microcalorimetric titrations of PP5A with MB (A) and PQ (B) in water at $298.15 \mathrm{~K}$

\section{$1.2 \mathrm{MB} / \mathrm{PP} 5 \mathrm{~A}$ 包合物对 $P Q$ 的竞争性荧光传感}

PP5A 与 MB 的主客体络合研究揭示了 PP5A 能与 $M B$ 形成 $1: 1$ 超分子包合物. 以 MB/PP5A 为探针, 进 一步研究其对 $\mathrm{PQ}$ 的竞争识别效应. 准确配制 0.2 $\mathrm{mmol} \cdot \mathrm{L}^{-1} \mathrm{MB} 、 0.5 \mathrm{mmol} \cdot \mathrm{L}^{-1} \mathrm{PP} 5 \mathrm{~A}$ 和 $10 \mathrm{mmol} \cdot \mathrm{L}^{-1} \mathrm{PQ}$, 分别移取 $250 \mu \mathrm{L} \mathrm{MB}$ 和 $500 \mu \mathrm{L}$ PP5A 置于 $10 \mathrm{~mL}$ 容量瓶 中, 然后逐渐加入 $5 \sim 2500 \mu \mathrm{L}$ 的 $\mathrm{PQ}$ 溶液, 超纯水定容、 摇匀并静置 $30 \mathrm{~min}$ 后测定其荧光光谱. 图 $3 \mathrm{~A}$ 为 $\mathrm{MB} / \mathrm{PP} 5 \mathrm{~A}$ 对 $\mathrm{PQ}$ 的苂光滴定曲线, 由图 3A 可知, 随着 $\mathrm{PQ}$ 的不断加入, MB/PP5A 的荧光强度不断增强直至恢 复, 说明加入的 PQ 竞争进入了 PP5A 的空腔中, MB 则 从 PP5A 的空腔中脱离出来, 从而使 MB/PP5A 体系的苂 光强度逐渐恢复. 在一定浓度范围内, 苂光强度与 $P Q$ 浓度具有良好的线性拟合关系. 如图 3B 所示, 直线方 程为 $y=3.47 \times 10^{6} x+117.19, R=0.9961 . \mathrm{MB} / \mathrm{PP} 5 \mathrm{~A}$ 对

表 $1 \mathrm{MB}$ 与不同大环化合物的结合常数对比

Table 1 Comparison of binding constants between MB and different macrocyclic compounds

\begin{tabular}{|c|c|c|c|c|c|}
\hline Host & Guest & Stoichiometric ratio & Binding constant & Solution conditions & Ref. \\
\hline CB7 & MB & $1: 1$ & $3.60 \times 10^{5}\left(\mathrm{~L} \cdot \mathrm{mol}^{-1}\right)$ & $\mathrm{pH}=3, \mathrm{HCl}$ aqueous solution & {$[44]$} \\
\hline CB7 & MB & $1: 1$ & $(4.2 \pm 0.3) \times 10^{4}\left(\mathrm{~L} \cdot \mathrm{mol}^{-1}\right)$ & $\mathrm{pH}=4.7, \mathrm{NaOAc}-\mathrm{HOAc}$ buffer solution & {$[45]$} \\
\hline CB7 & MB & $1: 1$ & $(1.26 \pm 0.28) \times 10^{7}\left({\left.\mathrm{~L} \cdot \mathrm{mol}^{-1}\right)}^{-1}\right.$ & $\mathrm{pH}=5.5$, Water solution & {$[46]$} \\
\hline CB8 & MB & $1: 2$ & $(1.06 \pm 0.53) \times 10^{16}\left(\mathrm{~mol} \cdot \mathrm{L}^{-1}\right)^{-2}$ & $\mathrm{pH}=5.5$, Water solution & {$[46]$} \\
\hline $\mathrm{SC} 4 \mathrm{~A}$ & MB & $1: 1$ & $1.41 \times 10^{5}\left({\left.\mathrm{~L} \cdot \mathrm{mol}^{-1}\right)}^{-1}\right.$ & $\mathrm{pH}=7.5$, Water solution & [47] \\
\hline SC6A & MB & $1: 1$ & $6.61 \times 10^{5}{\mathrm{~L} \cdot \mathrm{mol}^{-1}}^{-1}$ & $\mathrm{pH}=7.5$, Water solution & [47] \\
\hline$\beta C D$ & MB & $1: 1$ & $4.46 \times 10^{3}\left(\mathrm{~L} \cdot \mathrm{mol}^{-1}\right)$ & $\mathrm{pH}=7.2$, Tris- $\mathrm{HCl}$ buffer solution & [48] \\
\hline WP6A & MB & $1: 1$ & $(1.15 \pm 0.30) \times 10^{7}\left(\mathrm{~L} \cdot \mathrm{mol}^{-1}\right)$ & Water solution & [49] \\
\hline PP5A & MB & $1: 1$ & $(1.98 \pm 0.501) \times 10^{4}\left({\left.\mathrm{~L} \cdot \mathrm{mol}^{-1}\right)}^{-1}\right.$ & Water solution & This work \\
\hline
\end{tabular}




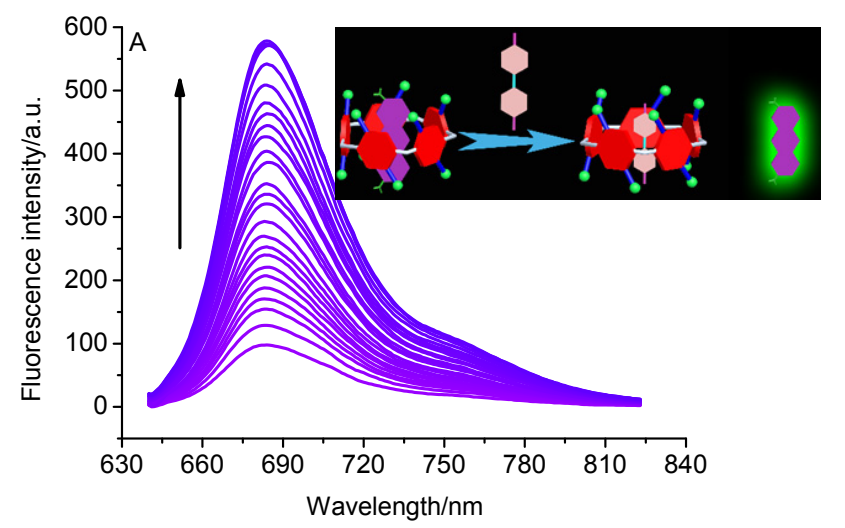

该传感器的检测限也低于部分方法, 说明 MB/PP5A 系 统检测 PQ 具有较为理想的检出限.

\subsection{PP5A 与 $P Q 、 M B$ 的核磁共振及紫外-可见吸收光 谱}

利用核磁共振氢谱研究了 PP5A 与 $\mathrm{PQ} 、 \mathrm{MB}$ 的相互 作用(图 4). 结果表明, 等物质的量的 PP5A 与 MB 发生 络合作用后, $M B$ 的 $H_{A} 、 H_{B}$ 和 $H_{D}$ 均向低场移动, 且 $H_{A}$ 、 $H_{B}$ 和 $H_{D}$ 的峰强度显著减弱, $H_{A}$ 和 $H_{B}$ 的双重峰消失, $H_{C}$ 的质子信号被屏蔽. 向 MB/PP5A 体系中加入等物质的 量的 $\mathrm{PQ}$ 时, $\mathrm{PQ}$ 与 $\mathrm{MB} / \mathrm{PP} 5 \mathrm{~A}$ 体系发生竞争络合作用, $\mathrm{PQ}$ 与 PP5A 发生络合, MB 从 PP5A 的空腔中脱离, MB 的

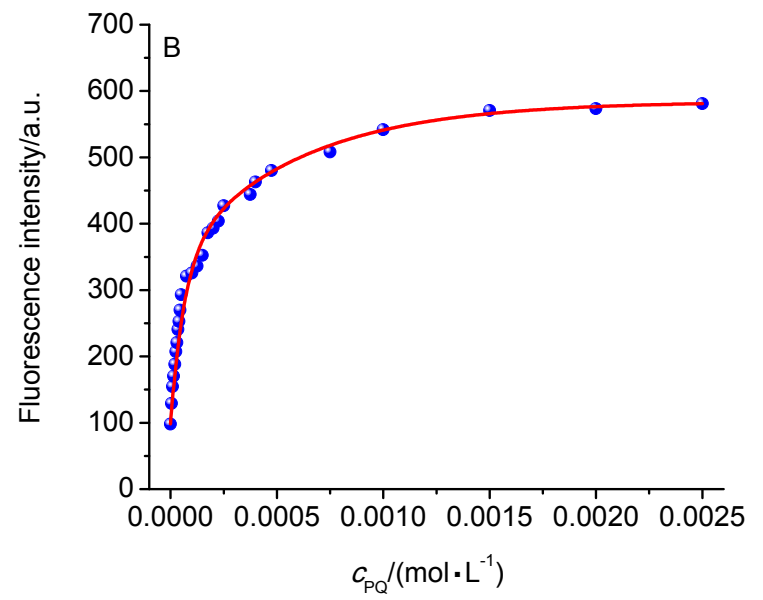

图 $3 \mathrm{MB} / \mathrm{PP} 5 \mathrm{~A}$ 包合物与 $\mathrm{PQ}$ 的苂光滴定图(A)以及 $\mathrm{MB} / \mathrm{PP} 5 \mathrm{~A}$ 包合物荧光强度与 $P Q$ 浓度的关系曲线 $(B)$

Figure 3 Fluorescence titration diagram of MB/PP5A inclusion complex (A) and the relationship between the fluorescence intensity of MB/PP5A inclusion complex and PQ concentration (B)

$\mathrm{PQ}$ 的最低检出限为 $3.6 \times 10^{-7} \mathrm{~mol} \cdot \mathrm{L}^{-1}(S / N=3)^{[50]}$. 结 果表明, MB/PP5A 对 PQ 的检测灵敏度较高, 可用作荧 光探针, 定量检测水溶液中的 $P Q$. 表 2 列举了检测 $P Q$ 的不同方法、线性范围以及检出限, 从表 2 中可以看出, 检测 PQ 的方法较为丰富, 但与其他报道相比较, MB/PP5A 检测系统具有操作简便、制备快捷的优点, 且 $\mathrm{H}_{\mathrm{A}}$ 和 $\mathrm{H}_{\mathrm{B}}$ 的双重峰信号恢复, 且 $\mathrm{H}_{\mathrm{D}}$ 的质子信号也明显恢 复.

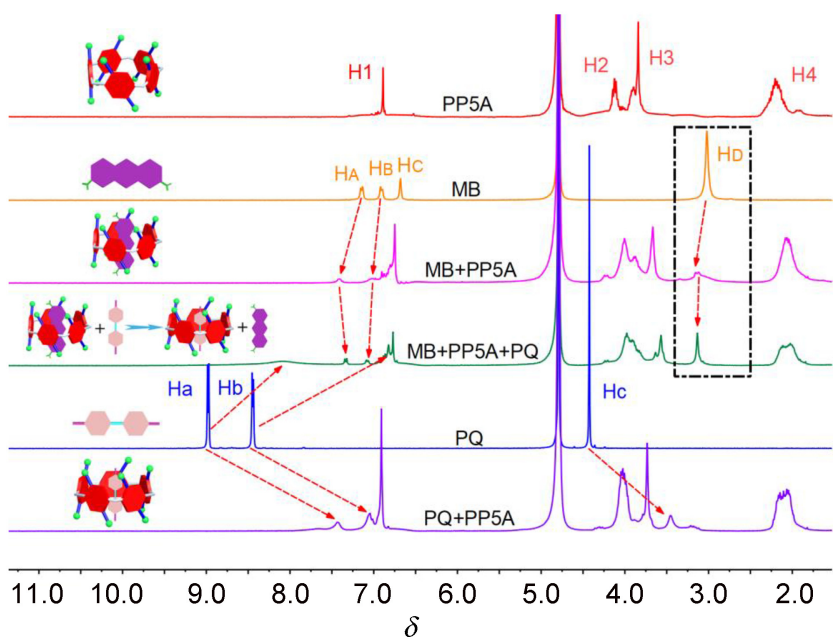

图 $4 \mathrm{PP} 5 \mathrm{~A}$ 与 $\mathrm{PQ} 、 \mathrm{MB}$ 的核磁共振氢谱 $\left(400 \mathrm{MHz}, \mathrm{D}_{2} \mathrm{O}\right)$

Figure $4{ }^{1} \mathrm{H}$ NMR spectra of PP5A with PQ and MB (400 $\left.\mathrm{MHz}, \mathrm{D}_{2} \mathrm{O}\right)$

PP5A $\left(20 \mathrm{mmol} \cdot \mathrm{L}^{-1}\right), \mathrm{MB}\left(20 \mathrm{mmol} \cdot \mathrm{L}^{-1}\right), \mathrm{MB} / \mathrm{PP} 5 \mathrm{~A}\left(20 \mathrm{mmol} \cdot \mathrm{L}^{-1}\right.$ $\left.\mathrm{MB}+20 \mathrm{mmol} \cdot \mathrm{L}^{-1} \mathrm{PP} 5 \mathrm{~A}\right), \mathrm{MB} / \mathrm{PP} 5 \mathrm{~A}+\mathrm{PQ}\left(20 \mathrm{mmol} \cdot \mathrm{L}^{-1} \mathrm{MB}+20\right.$ $\left.\mathrm{mmol} \cdot \mathrm{L}^{-1} \mathrm{PP} 5 \mathrm{~A}+20 \mathrm{mmol} \cdot \mathrm{L}^{-1} \mathrm{PQ}\right), \mathrm{PQ}\left(20 \mathrm{mmol} \cdot \mathrm{L}^{-1}\right), \mathrm{PQ} / \mathrm{PP} 5 \mathrm{~A}(20$ $\left.\mathrm{mmol} \cdot \mathrm{L}^{-1} \mathrm{PQ}+20 \mathrm{mmol} \cdot \mathrm{L}^{-1} \mathrm{PP} 5 \mathrm{~A}\right)$. The cartoon illustration is consistent with the corresponding structure in Scheme 1

表 2 PQ 的定量检测方法比较

Table 2 Comparison of different methods for quantitative detection of PQ

\begin{tabular}{|c|c|c|c|c|}
\hline Material & Method & $\begin{array}{c}\text { Linear range/ } \\
\left(\mu \mathrm{mol} \cdot \mathrm{L}^{-1}\right)\end{array}$ & $\begin{array}{c}\text { Detection limit/ } \\
\left(\mu \mathrm{mol} \cdot \mathrm{L}^{-1}\right)\end{array}$ & Ref. \\
\hline Phosphate salt pillar[5]arene/Graphene oxide & Fluorescence spectroscopy & $0.01 \sim 50.0$ & 0.0035 & {$[30]$} \\
\hline Phosphate salt pillar[6]arene/Graphene oxide & Fluorescence spectroscopy & $\begin{array}{l}0.2 \sim 2 \\
2 \sim 18\end{array}$ & 0.06 & {$[31]$} \\
\hline Citrate-coated silver nanoparticles & UV spectrum & $0.19 \sim 195.0$ & 0.19 & {$[51]$} \\
\hline Screen-printed carbon electrode & Differential pulse voltammetry & $0.54 \sim 4.3$ & 0.17 & {$[52]$} \\
\hline Pyranine & Fluorescence spectroscopy & $1.0 \sim 20.0$ & 0.20 & {$[53]$} \\
\hline Emitting diode-light dependent resistor & Flow injection colorimetric system & $0.78 \sim 38.9$ & 0.58 & {$[54]$} \\
\hline Squaraine and surfactants & Fluorescence spectroscopy & - & 0.37 & {$[55]$} \\
\hline MB/PP5A & Fluorescence spectroscopy & $1.0 \sim 50.0$ & 0.36 & This work \\
\hline
\end{tabular}


PP5A 与 $\mathrm{PQ}$ 发生络合作用后, $\mathrm{PQ}$ 的 $\mathrm{Ha} 、 \mathrm{Hb}$ 和 $\mathrm{Hc}$ 质子 均明显向高场方向移动. 上述核磁现象结果说明, 在水 溶液中 $\mathrm{MB}$ 和 $\mathrm{PQ}$ 均能与 PP5A 发生络合作用, PP5A 对 $\mathrm{PQ}$ 的络合能力强于 $\mathrm{MB}$.

此外, 通过紫外-可见吸收光谱实验也证实了 PP5A 与 $\mathrm{PQ} 、 \mathrm{MB}$ 之间主-客体络合物的形成(图 5), 原本呈无 色溶液的 PP5A 和 PQ 等物质的量混合后呈现淡黄色, 而深蓝色的 MB 溶液在加入等物质的量的 PP5A 溶液后 呈现出颜色变淡的现象, 表明主-客体之间存在电荷转 移相互作用. PP5A 与 PQ、MB 混合溶液的紫外-可见吸 收光谱显示出典型的电荷转移特征吸收峰, 说明富电性 的 PP5A 和缺电性的 PQ、MB 之间形成主-客体络合物, 且两者之间存在电荷转移相互作用. 分析原因是主体 PP5A 拥有富电子空腔, 与 PQ 和 MB 形成包合物时也存 在阳离子 $\pi$ 和 $\mathrm{C}-\mathrm{H}-\pi$ 等相互作用.
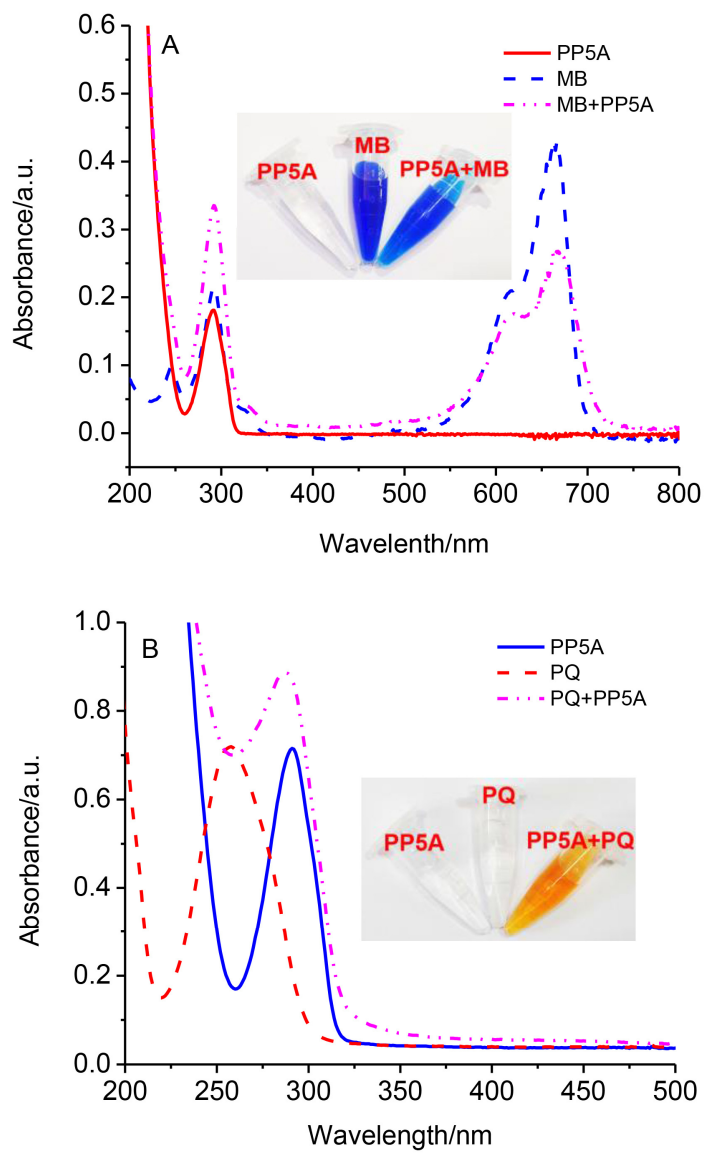

图 $5 \mathrm{PP} 5 \mathrm{~A}$ 与 $\mathrm{MB} 、 \mathrm{PQ}$ 的紫外-可见吸收光谱

Figure 5 UV-vis spectra of PP5A with $\mathrm{MB}$ and $\mathrm{PQ}$ (A) PP5A $\left(0.015 \mathrm{mmol} \cdot \mathrm{L}^{-1}\right), \mathrm{MB}\left(0.015 \mathrm{mmol} \cdot \mathrm{L}^{-1}\right), \mathrm{MB} / \mathrm{PP} 5 \mathrm{~A}(0.015$ $\left.\mathrm{mmol} \cdot \mathrm{L}^{-1} \mathrm{MB}+0.015 \mathrm{mmol} \cdot \mathrm{L}^{-1} \mathrm{PP} 5 \mathrm{~A}\right)$; (B) PP5A $\left(0.04 \mathrm{mmol} \cdot \mathrm{L}^{-1}\right) 、 \mathrm{PQ}$ $\left(0.04 \mathrm{mmol} \cdot \mathrm{L}^{-1}\right) 、 \mathrm{PQ} / \mathrm{PP} 5 \mathrm{~A}\left(0.04 \mathrm{mmol} \cdot \mathrm{L}^{-1} \mathrm{PQ}+0.04 \mathrm{mmol} \cdot \mathrm{L}^{-1} \mathrm{PP} 5 \mathrm{~A}\right)$

\section{4 动力学测试}

通过动力学测试探究了 $\mathrm{MB} / \mathrm{PP} 5 \mathrm{~A}$ 络合体系及 MB/PP5A 识别 PQ 时荧光强度与时间的变化关系. 移取
$250 \mu \mathrm{L} 0.2 \mathrm{mmol} \cdot \mathrm{L}^{-1}$ 的 $\mathrm{MB}$ 溶液和 $500 \mu \mathrm{L} 0.5 \mathrm{mmol} \cdot \mathrm{L}^{-1}$ 的 PP5A 溶液于 $10 \mathrm{~mL}$ 容量瓶中, 移入 PP5A 的瞬间开 始计时，定容摇匀后倒入苂光比色典, 1 min 时测定荧光 强度，随后每间隔 $1 \mathrm{~min}$ 测定一次苂光强度，连续测定 $30 \mathrm{~min}$. 移取 $250 \mu \mathrm{L} 0.2 \mathrm{mmol} \cdot \mathrm{L}^{-1}$ 的 $\mathrm{MB}$ 溶液、 $500 \mu \mathrm{L}$ $0.5 \mathrm{mmol} \cdot \mathrm{L}^{-1}$ 的 PP5A 溶液和 $250 \mu \mathrm{L} 10 \mathrm{mmol} \cdot \mathrm{L}^{-1}$ 的 PQ 溶液，移入 PQ 的瞬间开始计时，测试方法同 MB/PP5A 络合体系. 测定结束后以苂光强度为纵坐标，响应时间 为横坐标绘制动力学曲线(图 6). 从图 6A 中可以看出, 当 PP5A 加入到 $M B$ 溶液中时, $M B$ 在 $684 \mathrm{~nm}$ 处的苂光 强度瞬间降低，说明 PP5A 和 MB 的络合是在瞬间完成 的, 不具备时间响应. 而 MB/PP5A 体系对 PQ 的识别同 样不具时间响应，几乎在瞬间就完成响应(图 6B).
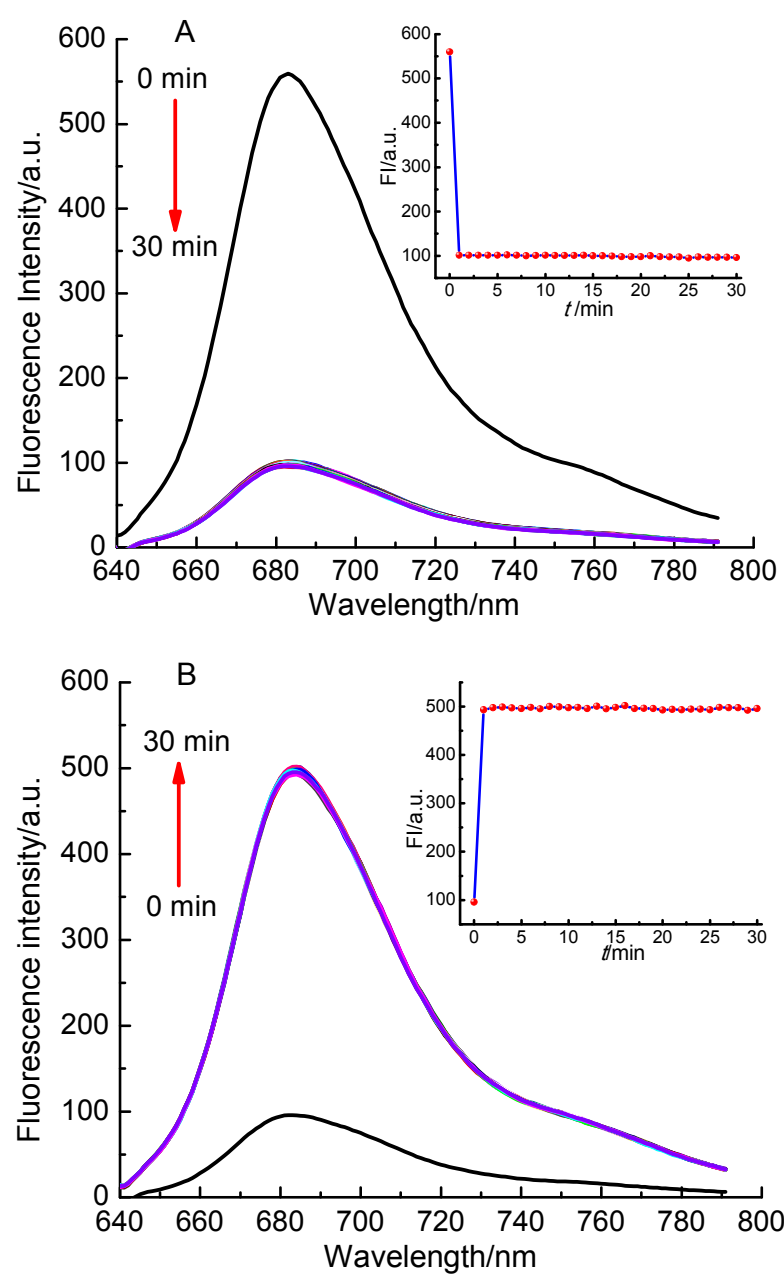

图 $6 \mathrm{MB} / \mathrm{PP} 5 \mathrm{~A}$ 络合体系 $(\mathrm{A})$ 及 $\mathrm{MB} / \mathrm{PP} 5 \mathrm{~A}$ 识别 $\mathrm{PQ}(\mathrm{B})$ 的动力 学曲线

Figure 6 Kinetic curves of MB/PP5A complex system (A) and MB/PP5A recognition PQ (B)

\section{$1.5 \mathrm{pH}$ 的影响}

$\mathrm{pH}$ 是影响苂光传感的重要因素, 为了讨论 $\mathrm{MB} /$ PP5A 在酸碱介质中的实用性，研究了 $\mathrm{pH}$ 对苂光强度的 
影响. 使用 $0.1 \mathrm{~mol} \cdot \mathrm{L}^{-1}$ 的 $\mathrm{HCl}$ 和 $\mathrm{NaOH}$ 调节出不同 $\mathrm{pH}$ 范围(1 13)的水溶液. 准确配制 $0.2 \mathrm{mmol} \cdot \mathrm{L}^{-1} \mathrm{MB} 、 0.5$ $\mathrm{mmol} \cdot \mathrm{L}^{-1} \mathrm{PP} 5 \mathrm{~A}$ 和 $10 \mathrm{mmol} \cdot \mathrm{L}^{-1} \mathrm{PQ}$, 分别移取 $250 \mu \mathrm{L}$ $\mathrm{MB}$ 和 $500 \mu \mathrm{L}$ PP5A 于 $10 \mathrm{~mL}$ 容量瓶中, 然后分别使用 不同 $\mathrm{pH}$ 范围 $(1 \sim 13)$ 的水溶液定容、摇匀即得 MB/PP5A 体系的待测液; 分别移取 $250 \mu \mathrm{L} \mathrm{MB} 、 500 \mu \mathrm{L} \mathrm{PP} 5 \mathrm{~A}$ 和 $250 \mu \mathrm{L} P Q$ 于 $10 \mathrm{~mL}$ 容量瓶中, 然后使用不同 $\mathrm{pH}$ 范围 $(1 \sim 13)$ 的水溶液定容、摇匀即得 MB/PP5A 识别 PQ 体 系的待测液. 不同 $\mathrm{pH}$ 条件下 $\mathrm{MB} / \mathrm{PP} 5 \mathrm{~A}$ 及 $\mathrm{MB} / \mathrm{PP} 5 \mathrm{~A}$ 识 别 PQ 体系的荧光强度如图 7 所示. MB/PP5A 能在 $\mathrm{pH}$ 值为 $5 \sim 11$ 范围内稳定地识别 $P Q$, 该 $\mathrm{pH}$ 范围也是生理 条件下应用的必要条件 ${ }^{[56]}$. 当 $\mathrm{pH}$ 值低于 5 时, 大量 $\mathrm{H}^{+}$ 与 PP5A 竞争结合和 MB 的质子化抑制了它与 PP5A 的 络合. 当 $\mathrm{pH}$ 值大于 12 时, $\mathrm{OH}^{-}$与 $\mathrm{MB}$ 结合导致了负电 荷增加, 抑制了与 PP5A 的络合. 因此, MB/PP5A 能在 较宽的 $\mathrm{pH}$ 范围下高效稳定的检测 PQ.

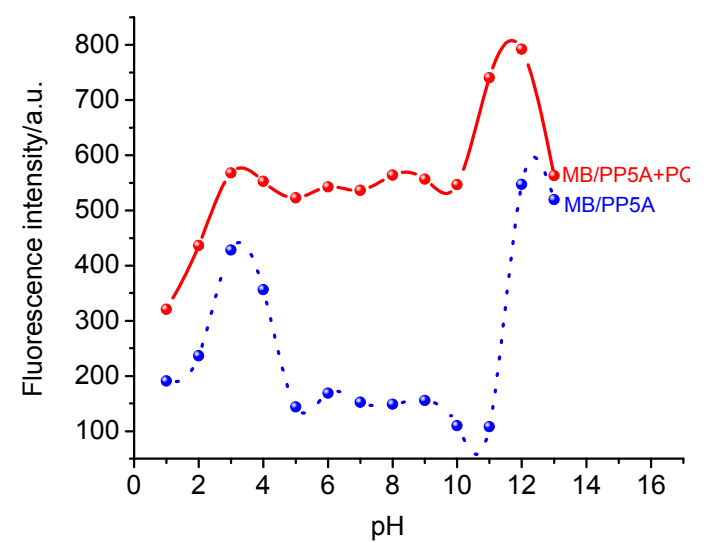

图 $7 \mathrm{pH}$ 对 $\mathrm{MB} / \mathrm{PP} 5 \mathrm{~A}$ 包合物荧光强度和 $\mathrm{PQ}$ 检测的影响曲线

Figure 7 Effect of $\mathrm{pH}$ on MB/PP5A inclusion complex fluorescence intensity and PQ detection

\section{6 抗干扰实验}

荧光探针对目标物的选择性识别程度决定了其实 际应用前景. 为了探究 MB/PP5A 探针在生物体环境中 检测 PQ 的应用, 通过 20 种氨基酸和 13 种常见阴离子 的荧光竞争性实验评价了 $\mathrm{MB} / \mathrm{PP} 5 \mathrm{~A}$ 探针对 $\mathrm{PQ}$ 的选择 性识别性能. 准确配制 $0.2 \mathrm{mmol} \cdot \mathrm{L}^{-1} \mathrm{MB} 、 0.5 \mathrm{mmol} \cdot \mathrm{L}^{-1}$ PP5A、 $10 \mathrm{mmol} \cdot \mathrm{L}^{-1} \mathrm{PQ} 、 10 \mathrm{mmol} \cdot \mathrm{L}^{-1} \mathrm{PQ} 1 、 10 \mathrm{mmol} \cdot$ $\mathrm{L}^{-1}$ 氨基酸(Ala、Val、Leu、Ile、Pro、Phe、Met、Tyr、 Asn、Gln、Lys、Arg、His、Asp、Glu、Trp)、10 mmol• $\mathrm{L}^{-1}$ 阴离子 $\left(\mathrm{F}^{-} 、 \mathrm{Cl}^{-} 、 \mathrm{Br}^{-} 、 \mathrm{I}^{-} 、 \mathrm{PO}_{4}^{3-} 、 \mathrm{HPO}_{3}^{2-} 、 \mathrm{HSO}_{3}^{-}\right.$、 $\left.\mathrm{SO}_{4}^{2-} 、 \mathrm{NO}_{2}^{-} 、 \mathrm{NO}^{3-} 、 \mathrm{~S}^{2-} 、 \mathrm{CO}_{3}^{2-} 、 \mathrm{HCO}_{3}^{-}\right) 、 10 \mathrm{mmol} \bullet$ $\mathrm{L}^{-1}$ 阳离子 $\left(\mathrm{Na}^{+} 、 \mathrm{Cu}^{2+} 、 \mathrm{~K}^{+} 、 \mathrm{Zn}^{2+} 、 \mathrm{Mn}^{2+} 、 \mathrm{Al}^{3+} 、 \mathrm{~Pb}^{2+} 、\right.$ $\left.\mathrm{Cs}^{2+} 、 \mathrm{Ag}^{+} 、 \mathrm{Fe}^{3+}\right) 、 10 \mathrm{mmol} \cdot \mathrm{L}^{-1}$ 葡萄糖和 $10 \mathrm{mmol} \cdot \mathrm{L}^{-1}$ 蔗糖. 分别移取 $250 \mu \mathrm{L} \mathrm{MB}$ 和 $500 \mu \mathrm{L}$ PP $5 \mathrm{~A}$ 于 $10 \mathrm{~mL}$ 容 量瓶中, 然后分别加入 $250 \mu \mathrm{L}$ 的不同氨基酸、 $\mathrm{PQ} 1$ 、阴
离子和阳离子, 定容摇匀后即得 $\mathrm{MB} / \mathrm{PP} 5 \mathrm{~A}$ 与氨基酸、 $\mathrm{PQ} 1$ 、阴离子、阳离子、葡萄糖和蔗糖的对照待测液. 分 别移取 $250 \mu \mathrm{L} \mathrm{MB} 、 500 \mu \mathrm{L}$ PP $5 \mathrm{~A}$ 和 $250 \mu \mathrm{L}$ PQ 于 $10 \mathrm{~mL}$ 容量瓶中, 然后分别加入 $250 \mu \mathrm{L}$ 的不同种氨基酸、PQ1、 阴离子、阳离子、阳离子、葡萄糖和蔗糖，定容摇勺后 即得待测液. MB/PP5A 探针识别 PQ 的抗干扰性能测试 如图 8 所示. 从图 $8 \mathrm{~A}$ 可看出, 只有 $\mathrm{PQ}$ 的加入才能使 MB/PP5A 探针的苂光发生显著增强, Gln 与 Asp 虽然对 MB/PP5A 探针存在着一定的竞争作用, 但 PQ 对 $\mathrm{MB} / \mathrm{PP} 5 \mathrm{~A}$ 探针的苂光增效作用更为强烈. 此外, 如图 $8 \mathrm{~B}$ 所示, 在 20 种氨基酸、 13 种阴离子、 10 种阳离子、 1 种类似物、葡萄糖和蔗糖的存在下, PQ 也依然能使 $\mathrm{MB} / \mathrm{PP} 5 \mathrm{~A}$ 探针的苂光发生显著增强. 显然, MB/PP5A 探针对 PQ 的选择性识别具有较为良好的抗干扰性能, 该探针也有望应用于生物体环境下 PQ 的检测识别.
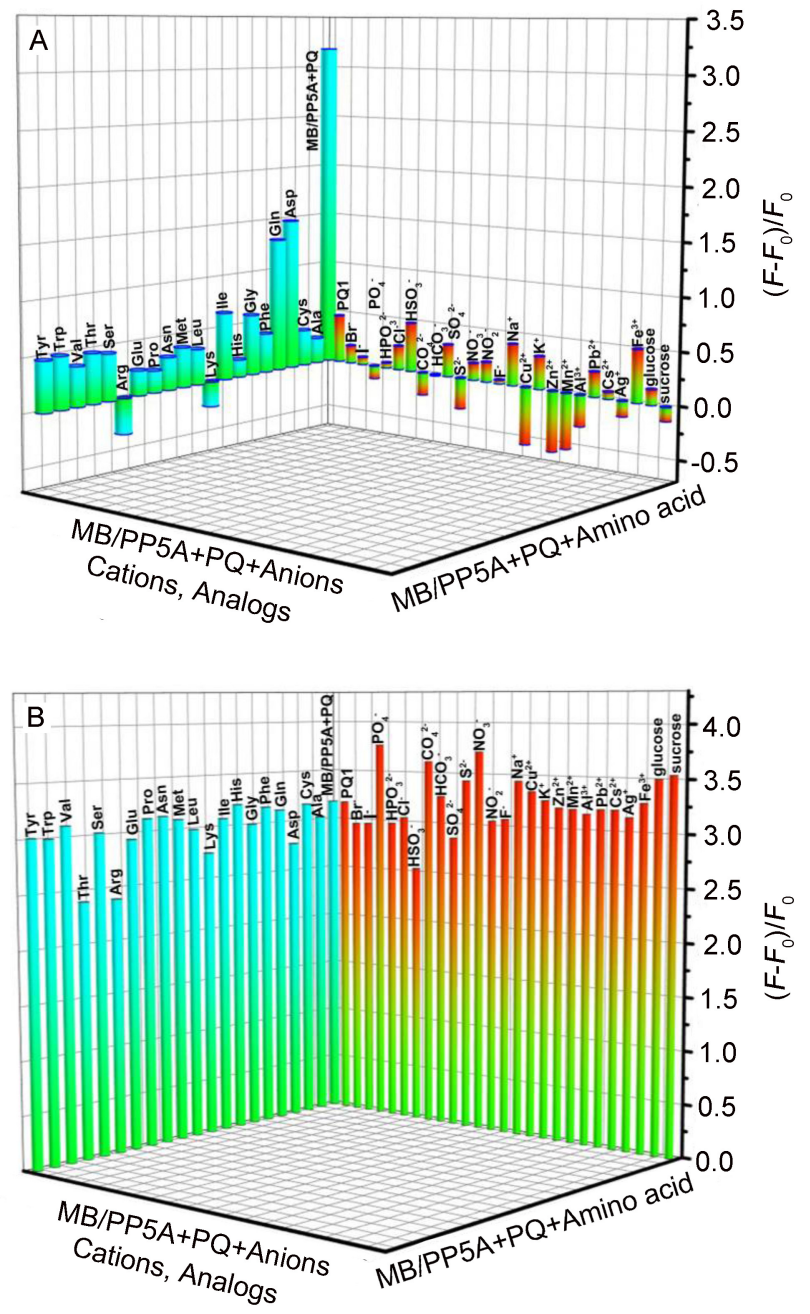

图 8 阴离子和氨基酸对识别体系的影响

Figure 8 Effect of anions and amino acids on the recognition system

(A) $\mathrm{MB} / \mathrm{PP} 5 \mathrm{~A}+$ amino acids or anions; (B) $\mathrm{MB} / \mathrm{PP} 5 \mathrm{~A}+\mathrm{PQ}+$ amino acids, anions, cations or analogs 


\section{7 分子对接分析}

将均方根偏差小于 $0.2 \mathrm{~nm}$ 的构像归为一簇进行聚 类分析, 得到的最稳定主-客体对接模式如图 9 所示. 显 然, 没有出现 $\mathrm{PQ}$ 或 $\mathrm{MB}$ 分子脱离 PP5A 空腔的结果, 说 明 PP5A 与 $\mathrm{MB} 、 \mathrm{PQ}$ 之间均可形成包合物 ${ }^{[57]}$. PP5A 与 $\mathrm{PQ}$ 经 50 次对接计算后只形成一簇对接构象, 图 9 (PQ-1、PQ-2) 为 PP5A 与 PQ 形成的最稳定主-客体对接 模式, PP5A 与 $\mathrm{PQ}$ 的结合自由能 $\left(\Delta G_{\text {bind }}\right)$ 为 -16.55 $\mathrm{kJ} / \mathrm{mol}$. PP $5 \mathrm{~A}$ 与 MB 经 50 次对接计算后形成了两簇对 接构象, 第一簇构象为主要构象簇, 该簇共搜索了 42 个 构象结构, 簇中最优构象的 $\Delta G_{\text {bind }}$ 为 $57.20 \mathrm{~kJ} / \mathrm{mol}$, 而第 二簇构象为次要构象簇, 共搜索了 8 个构象结构, 该簇 中最优构象的 $\Delta G_{\text {bind }}$ 为 $64.97 \mathrm{~kJ} / \mathrm{mol}$, 与 PP $5 \mathrm{~A}$ 和 PQ 的 对接构象类似，二者的几何中心并没有重叠，分子均处 于 PP5A 空腔的一端. 客体分子斜靠于主体分子的内壁, 包合物中主-客体结合紧密使能量降低. PP5A 的空腔对 $\mathrm{PQ}$ 和 MB 分子均具有较好的空间匹配性. 此外, $\Delta G_{\text {bind }}$ 值越低说明主体与客体间的亲和力就越强 ${ }^{[58]}$. 因此, 与 $\mathrm{MB}$ 相比, PP5A 与 PQ 的具有更强的亲和力, 这与 ITC 和 ${ }^{1} \mathrm{H}$ NMR 的分析结果吻合.

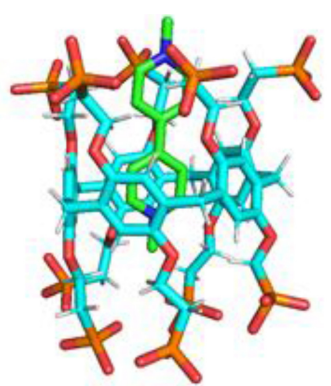

$P Q-1$

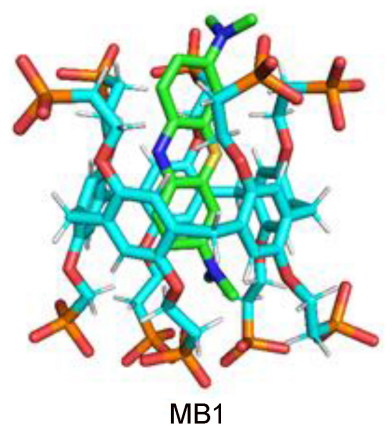

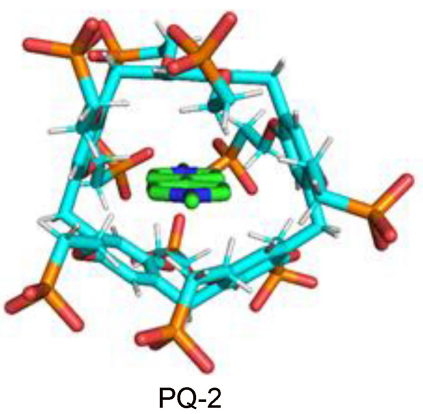

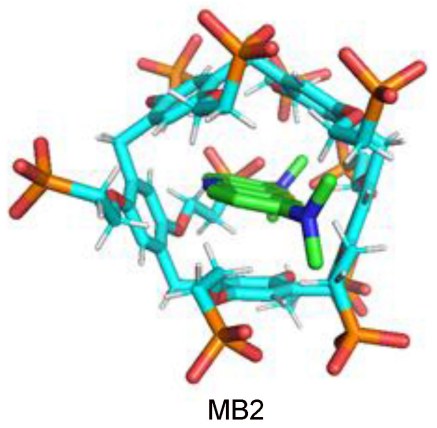

图 $9 \mathrm{PP} 5 \mathrm{~A}$ 与 $\mathrm{PQ} 、 \mathrm{MB}$ 的分子对接包合模式

Figure 9 Molecular docking conformation diagram of PP5A with PQ and MB

PQ-1 and MB-1 represent front view, and PQ-2 and MB-2 represent top view

\section{2 结论}

本文利用 PP5A 作为主体, MB 作为客体, 构筑了 $\mathrm{MB} / \mathrm{PP} 5 \mathrm{~A}$ 主-客体包合物, PP5A 与 MB 的主-客体络合
分析表明, MB 与 PP5A 能形成 $1: 1$ 包合物, 结合常数 为 $(1.98 \pm 0.501) \times 10^{4} \mathrm{~L} \cdot \mathrm{mol}^{-1}$, 且 MB 被 PP5A 包合后其 荧光发生淬灭. 进一步探索了 $\mathrm{MB} / \mathrm{PP} 5 \mathrm{~A}$ 包合物对 $\mathrm{PQ}$ 竞 争性荧光传感, 将 PQ 加入到 MB/PP5A 传感系统中, PQ 竞争进入 PP5A 的空腔中, 而 MB 从 PP5A 的空腔中脱 离导致苂光恢复. 通过 1 种类似物、20 种氨基酸、13 种阴离子、10 种金属阳离子、葡萄糖和蔗糖研究了 MB/PP5A 探针识别 PQ 时的抗干扰性能, 发现 MB/PP5A 探针能专一性的识别 PQ. 此外, MB/PP5A 苂 光传感系统可以在 $\mathrm{pH}=5 \sim 11$ 范围内有效识别 $P Q$. $\mathrm{MB} / \mathrm{PP} 5 \mathrm{~A}$ 苂光传感系统识别 PQ 的最低检测限为 $3.6 \times$ $10^{-7} \mathrm{mmol} \cdot \mathrm{L}^{-1}$, 可以有效灵地检测 $\mathrm{PQ}$. 综上所述, MB/PP5A 苂光传感系统对 PQ 的识别具有选择性强、制 备简便快捷、 $\mathrm{pH}$ 宽域测定和较为良好的抗干扰性能等 优点, 可为生物体及环境中 PQ 的传感检测提供基础.

\section{3 实验部分}

\section{1 仪器与试剂}

Aglient Cary Eclips 苂光分光光度计, Aglient 8453 紫外-可见分光光度计, Microcal VP-ITC 微量热计, Bruker Avance 400 核磁共振仪. 三氟化嗍乙醚、1, 4-二 (2-羟基乙氧基)苯、四溴化碳、三苯基膦、多聚甲醛、 亚磷酸三乙酯、四甲基溴硅烷、氨水、百草枯、亚甲基 蓝、葡萄糖(glucose) 和蔗糖(sucrose); 1 种百草枯类似物: 4,4'-联吡啶(PQ1)；20 种氨基酸: 丙氨酸(Ala)、傾氨酸 (Val)、亮氨酸(Leu)、异亮氨酸(Ile)、脯氨酸(Pro)、苯丙 氨酸(Phe)、蛋氨酸(Met)、甘氨酸(Tyr)、天冬酰胺(Asn)、 谷氨酰胺 $(\mathrm{Gln}) 、$ 赖氨酸 $(\mathrm{Lys}) 、$ 精氨酸 $(\mathrm{Arg}) 、$ 组氨酸( $\mathrm{His}) 、$ 天冬氨酸(Asp)、谷氨酸(Glu)和色氨酸(Trp); 13 种常见阴 离子钠盐: 澳化钠 $(\mathrm{NaBr})$ 、碘化钠 $(\mathrm{NaI})$ 、氟化钠 $(\mathrm{NaF})$ 、 氯化钠 $(\mathrm{NaCl})$ 、硫化钠 $\left(\mathrm{Na}_{2} \mathrm{~S}\right)$ 、硝酸钠 $\left(\mathrm{NaNO}_{3}\right)$ 、亚硝酸 钠 $\left(\mathrm{NaNO}_{2}\right)$ 、硫酸钠 $\left(\mathrm{Na}_{2} \mathrm{SO}_{4}\right)$ 、亚硫酸钠 $\left(\mathrm{Na}_{2} \mathrm{SO}_{3}\right)$ 、碳酸 钠 $\left(\mathrm{Na}_{2} \mathrm{CO}_{3}\right)$ 、碳酸氢钠 $\left(\mathrm{NaHCO}_{3}\right)$ 、磷酸钠 $\left(\mathrm{Na}_{3} \mathrm{PO}_{4}\right)$ 和磷 酸氢二钠 $\left(\mathrm{Na}_{2} \mathrm{HPO}_{4}\right) ; 10$ 种金属阳离子: 硫酸钠 $\left(\mathrm{Na}_{2} \mathrm{SO}_{4}\right)$ 、五水硫酸铜 $\left(\mathrm{CuSO}_{4} \cdot 5 \mathrm{H}_{2} \mathrm{O}\right)$ 、氯化钾 $(\mathrm{KCl})$ 、七 水硫酸锌 $\left(\mathrm{ZnSO}_{4} \cdot 7 \mathrm{H}_{2} \mathrm{O}\right)$ 、乙酸锰 $\left(\mathrm{CH}_{4} \mathrm{CH}_{6} \mathrm{MnO}_{4}\right)$ 、九水 硝酸铝 $\left(\mathrm{Al}\left(\mathrm{NO}_{3}\right)_{3} \cdot 9 \mathrm{H}_{2} \mathrm{O}\right)$ 、硝酸铅 $\left[\mathrm{Pb}\left(\mathrm{NO}_{3}\right)_{2}\right]$ 、碳酸铯 $\left(\mathrm{Cs}_{2} \mathrm{CO}_{3}\right)$ 、硝酸银 $\left(\mathrm{AgNO}_{3}\right)$ 、六水三氯化铁 $\left(\mathrm{FeCl}_{3} \bullet 6 \mathrm{H}_{2} \mathrm{O}\right)$ 均为分析纯试剂, 购于上海泰坦科技有限公司, 其余均 为市售分析纯试剂, 实验用水为超纯水.

\section{2 实验方法}

参照文献[59]合成 PP5A, 其路线如 Scheme 2 所示.

化合物 2 的合成: 称取化合物 1 (10.00 g, 50.4 $\mathrm{mmol}) 、 \mathrm{PPh}_{3}(32.0 \mathrm{~g}, 120 \mathrm{mmol})$ 于烧瓶中, 加入 $200 \mathrm{~mL}$ $\mathrm{CH}_{3} \mathrm{CN}$, 然后在氮气保护下逐滴加入 $\mathrm{CBr}_{4}(40.0 \mathrm{~g}, 120$ 

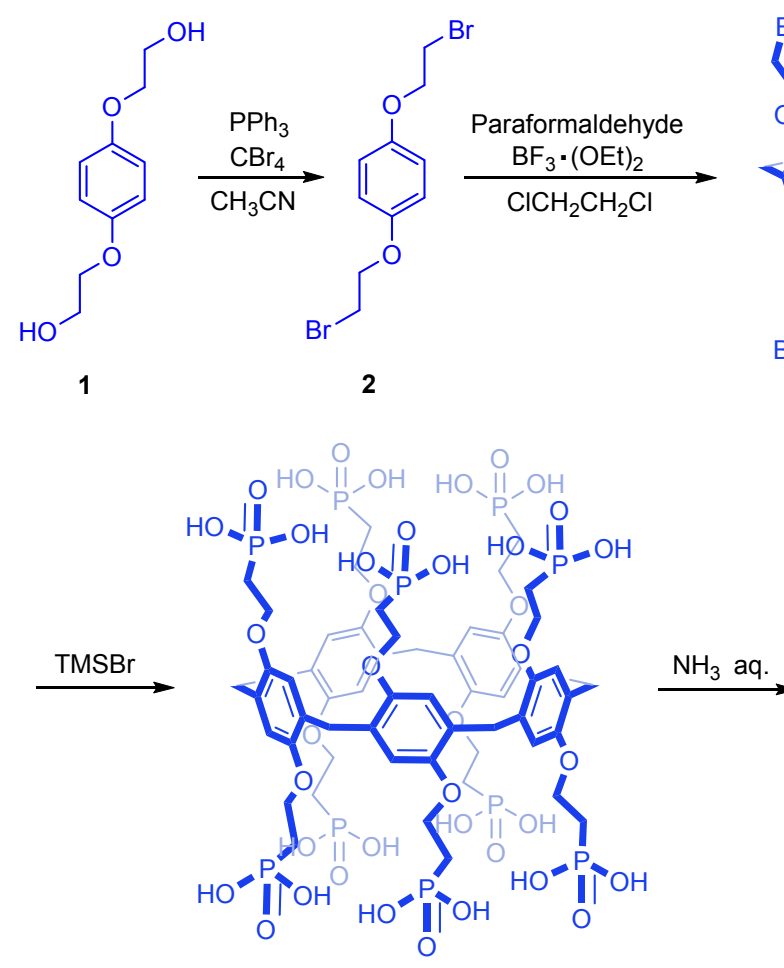

5
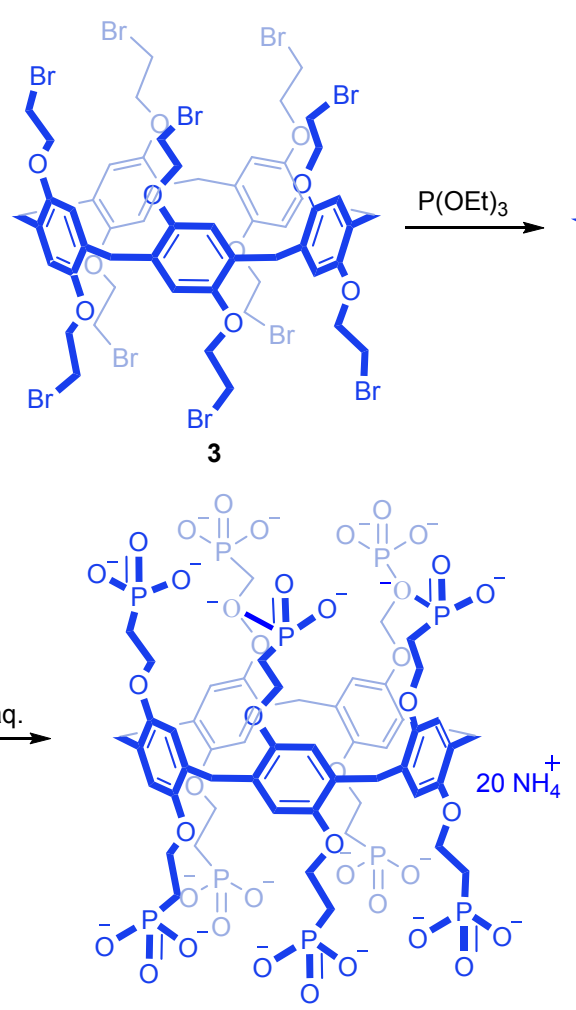

PP5A

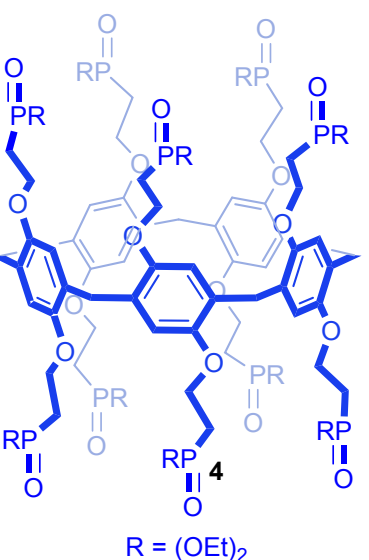

图式 2 PP5A 的合成路线

Scheme 2 Synthetic route of PP5A

$\mathrm{mmol})$ 的 $\mathrm{CH}_{3} \mathrm{CN}(50 \mathrm{~mL})$ 溶液, 室温反应 $5 \mathrm{~h}$ 后倒入冰 水, 得到大量白色晶体. 过滤后用石油醚/甲醇 $(V: V=$ $1:$ 1)洗涤, 得白色碎片状晶体 $214.4 \mathrm{~g}$, 产率 $92 \%$. m.p. $114 \sim 116{ }^{\circ} \mathrm{C}$ (文献值 ${ }^{[60]} 114 \sim 115{ }^{\circ} \mathrm{C}$ ).

化合物 3 的合成: 称取化合物 $2(1.62 \mathrm{~g}, 5 \mathrm{mmol})$ 、

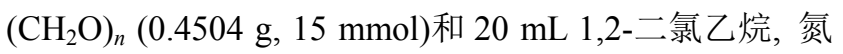
气保护下逐滴加入三氟化硼乙醚 $(0.70 \mathrm{~g}, 5 \mathrm{mmol})$, 反应 $6 \mathrm{~h}$ 后加水淬灭, 饱和食盐水洗涤 $(50 \mathrm{~mL} \times 2)$, 收集下层 有机相, 无水硫酸钠干燥, 减压浓缩, 柱层析纯化 $[V$ (石 油醚)：V(二氯甲烷) $=1 ：$ 1]得白色粉末状固体 $30.81 \mathrm{~g}$, 产率 50\%. m.p. 95 97 ${ }^{\circ} \mathrm{C}$ (文献值 ${ }^{[60]} 95 \sim 97{ }^{\circ} \mathrm{C}$ ).

化合物 $\mathbf{4}$ 的合成: 称取化合物 $\mathbf{3}(2.50 \mathrm{~g}, 1.49 \mathrm{mmol})$ 和亚磷酸三乙酯 $(24.71 \mathrm{~g}, 149 \mathrm{mmol})$, 在氮气氛围下 $165{ }^{\circ} \mathrm{C}$ 搅拌 $72 \mathrm{~h}$, 浓缩, 柱层析 $V$ (甲醇) : $V$ (乙酸乙 酯) $=1:$ 2) 得浅黄色油状物 $\mathbf{4} 3.02 \mathrm{~g}$, 产率 $90 \%$.

化合物 5 的合成: 在 $0{ }^{\circ} \mathrm{C}$, 氮气氛围下, 将 $\mathrm{TMSBr}$ $(14.25 \mathrm{~g}, 93.1 \mathrm{mmol})$ 加入到化合物 $4(3.0 \mathrm{~g}, 1.33 \mathrm{mmol})$ 的二氯甲烷溶液中, 然后在室温下搅拌 $72 \mathrm{~h}$. 反应结束 后减压蒸馏除去溶剂, 加入水 $(30 \mathrm{~mL})$ 继续摚拌 $30 \mathrm{~min}$, 浓缩干燥后得到浅黄色固体, 使用丙酮洗涤粗产物即得 白色固体 $51.75 \mathrm{~g}$, 产率 78\%. m.p. $>300{ }^{\circ} \mathrm{C}$.
化合物 PP5A 的合成: 将化合物 $5(500 \mathrm{mg}, 0.3$ $\mathrm{mmol})$ 中加入氨水 $(30 \%, 200 \mathrm{~mL})$ 搅拌 $12 \mathrm{~h}$ 后旋干即得淡 黄色固体 PP5A $603 \mathrm{mg}$, 产率 98\%. m.p.>300 ${ }^{\circ} \mathrm{C}$. PP5A 及其中间体经 NMR 表征, 谱图数据与文献[59]一致.

\section{3 分子对接模拟}

采用分子对接模拟进一步研究 PP5A 与 $M B 、 P Q$ 的 主一客体络合模式. PP5A、MB 和 PQ 的结构均通过 MM2 力场优化至能量最小值. 将 PP5A 设置为刚性受体分子, $M B 、 P Q$ 则作为配体分子允许柔性扭转. 设置 PP $5 A$ 受 体的格点间距盒子尺寸为: 25 点 $\times 25$ 点 $\times 40$ 点, 盒子中 心坐标为 $(-0.776,-0.016,-0.502)$, 网格间距为 $0.0375 \mathrm{~nm}, \mathrm{PP} 5 \mathrm{~A}$ 分子的几何中心即为盒子的几何中心. 基于拉马克遗传算法(Lamarckian, GA 4.2)将 $\mathrm{MB} 、 \mathrm{PQ}$ 分 别对接到 PP5A 的空腔中, 执行 50 次构象搜索, 最大评 估数为 $2.5 \times 10^{7}$, 其他参数使用默认值. 对接完成后, 将均方根偏差小于 $0.2 \mathrm{~nm}$ 的构像归为一簇进行聚类分 析. 分子对接采用 AutoDock 4.2 程序 ${ }^{[61]}$ 完成.

辅助材料(Supporting Information) 化合物 $2 \sim 5$ 和 PP5A 的核磁表征数据. 这些材料可以免费从本刊网站 (http://sioc-journal.cn/)上下载. 


\section{References}

[1] Desipio, M. M.; Thorpe, R.; Saha, D. Optik 2018, 172, 1047.

[2] Siangproh, W.; Somboonsuk, T.; Chailapakul, O.; Songsrirote, K. Talanta 2017, 174, 448.

[3] Hu, X.; Liang, Y.-Y.; Zhao, H.-Y.; Zhao, M. Int. Immunopharmacol. 2019, 67, 231

[4] Gao, C.; Huang, Q.-X.; Lan, Q.-P.; Feng, Y.; Tang, F.; Hoi, M. P. M.; Zhang;, J.-X.; Lee S. M. Y.; Wang, R.-B. Nat. Commun. 2018, 9, 2967.

[5] Yu, G.-C.; Zhou, X.-Y.; Zhang, Z.-B.; Han, C.-Y.; Mao, Z.-W.; Gao, C.-Y.; Huang, F.-H. J. Am. Chem. Soc. 2012, 134, 19489.

[6] Wang, K.; Guo, D.-S.; Zhang, H.-Q.; Li, D.; Zheng, X-L.; Liu, Y. J. Med. Chem. 2009, 52, 6402 .

[7] Zhao, Z.-Z.; Zhang, F.-W.; Zhang, Z.-P. Spectrochim. Acta A 2018 , 199, 96.

[8] Tcheumi, H. L.; Tassontio, V. N.; Tonle, I. K.; Ngameni, E. Appl. Clay Sci. 2019, 173, 97.

[9] Rai, M. K.; Das, J. V.; Gupta, V. K. Talanta 1997, 45, 343.

[10] Lu, T.; Birke, R. L.; Lombardi, J. R. Langmuir 1986, 2, 305.

[11] Kambhampati, I.; Roinestad, K. S.; Hartman, T. G.; Rosen, J. D.; Fukuda, E. K.; Lippincott, R. L.; Rosen, R. T. J. Chromatogr. A 1994, 688, 67

[12] Gao, L.; Liu, J.-T.; Wang, C.-Y.; Liu, G.-J.; Niu, X.-D.; Shu, C.-X.; Zhu, J. J. Chromatogr. B 2014, 944, 136.

[13] Tomková, H.; Sokolová, R.; Opletal, T.; Kučerová, P.; Kučera, L.; Součková, J.; Skopalová, J.; Barták, P. J. Electroanal. Chem. 2018, $821,33$.

[14] Vinner, E.; Stievenart, M.; Humbert, L.; Mathieu, D. Biomed. Chromatogr. 2001, 15, 342.

[15] Usui, K.; Minami, E.; Fujita, Y.; Kobayashi, H.; Hanazaw, T.; Kamijo, Y.; Funayama, M. J. Pharmacol. Toxicol. Methods 2019, 100, 106610.

[16] Yang, Y.-H.; Yang, J.-L.; Du, Y.; Li C.-H.; Wei K.-K.; Lu, J.-J.; Chen, W.; Yang, L.-J. ACS Omega 2019, 4, 17741.

[17] Du, T.; Yuan, W.; Zhao, Z. Y.; Liu, S. M. Chem. Commun. 2019, 55, 3658.

[18] Xie, Z.-P.; Lei, J.-L.; Yang, M.-F.; Li, Y.-J.; Geng, X.-H.; Liu, S.-M.; Wang, J.-H. Biosens. Bioelectron. 2019, 127, 200.

[19] Chen, Y.; Huang, F.-H.; Li, Z.-T.; Liu, Y. Sci. China Chem. 2018, $61,979$.

[20] Huo, B.-C.; Li, B.; Su, H.; Zeng, X. Q.; Xu, K.-D.; Cui, L. Chin. J. Org. Chem. 2019, 39, 1990 (in Chinese). (霍博超, 李斌, 苏杭, 曾宪强, 徐凯迪, 崔雷, 有机化学, 2019, 39, 1990.)

[21] Sun, C.-L.; Teng, K.-X.; Niu, L.-Y.; Chen, Y.-Z.; Yang, Q.-Z. Acta Chim. Sinica 2018, 76, 779 .

[22] Li, P.-Y.; Chen, Y.; Liu, Y. Chin. Chem. Lett. 2019, 30, 1190.

[23] Wang, M.-J.; Du, X.-S.; Tian, H.-S.; Jia, Q.; Deng, R.; Cui, Y.-H.; Wang, C. Y; Kamel, M. Chin. Chem. Lett. 2019, 30, 345.

[24] Li, Z.-T.; Yang, J.; Huang, F.-H. Chin. J. Chem. 2018, 36, 59.

[25] Jie, K.-C.; Zhou, Y.-J.; Li, E.-R.; Huang, F.-H. Acc. Chem. Res. 2018, 51, 2064.

[26] Shao, W.; Liu, X.; Wang, T.-T.; Hu, X.-Y. Chin. J. Org. Chem. 2018, 38, 1107 (in Chinese) (绍为, 刘昕, 王婷婷, 胡晓玉, 有机化学, 2018, 38, 1107.)

[27] Pian, M. D.; Schalley, C. A.; Fabris, F.; Scarso, A. Org. Chem. Front. 2019, 6, 1044.

[28] Cao, D.-R.; Herbert, M. Chin. Chem. Lett. 2019, 30, 1758.

[29] Zhang, Z.-B.; Sun, K.-C.; Li, S.-J.; Yu, G.-C. Chin. Chem. Lett. 2019, 30, 957

[30] Tan, X.-P.; Wu, Y.; Yu, S.; Zhang, T.-Y.; Tian, H.-X.; He, S.-H.; Zhao, A.-N.; Chen, Y.-W.; Gou, Q. Talanta 2019, 195, 472.

[31] Qian, X. C.; Zhou, X. J.; Gao, W.; Li, J.; Ran, X.; Du, G. B.; Yang, L. Microchem. J. 2019, 150, 104203

[32] Li, H. Chen, D.-X.; Sun, Y.-L.; Zheng, Y.-B.; Tan, L.-L.; Weiss, P.-S.; Yang, Y.-W. J. Am. Chem. Soc. 2013, 135, 1570.

[33] Wang, X.; Liu, Z. J.; Hill, E. H.; Zheng, Y. B.; Guo, G. Q.; Wang,
Y.; Weiss, P. S.; Yu, J. H.; Yang, Y. W. Matter 2019, 1, 848.

[34] Sun, S.-G.; Li, F.-S.; Liu, F.-Y.; Wang, J.-T.; Peng, X.-J. Sci. Rep. 2014, 4, 3570 .

[35] Mao, X.-W.; Liu, T.; Bi, J.-H.; Luo, L.; Tian, D.-M.; Li, H.-B. Chem. Commun. 2016, 52, 4385 .

[36] Ogoshi, T.; Kanai, S.; Fujinami, S.; Yamagishi, T. A.; Nakamoto, Y. J. Am. Chem. Soc. 2008, 130, 5022 .

[37] Shi, H.-X.; Cheng, X.-B.; Lin, Q.; Yao, H.; Zhang, Y.-M.; Wei, T.-B. Chin. J. Org. Chem. 2018, 38, 1718 (in Chinese). (师海雄, 程晓斌, 林奇, 姚虹, 张有明, 魏太保, 有机化学, 2018, 38, 1718.)

[38] Li, Z.-Y.; Hou, N.-N.; Shao, W.; Xiao, S.-J.; Lin, C.; Wang, L.-Y. Chin. J. Org. Chem. 2018, 38, 2002 (in Chinese) (李臻益, 候娜娜, 邵为, 肖守军, 林晨, 王乐勇, 有机化学, 2018, 38, 2002.)

[39] Wang, X.; Wu, J.-R.; Liang, F.; Yang, Y.-W. Org. Lett. 2019, 21, 5215.

[40] Ma, X.-Q.; Wang, Y.; Wei, T.-B.; Qi, L.-H.; Jiang, X.-M.; Ding, J.-D.; Zhu, W.-B.; Yao, H.; Zhang, Y.-M.; Lin, Q. Dyes Pigm. 2019, $164,279$.

[41] Chen, J.-F.; Liu, X.; Han, B.-B.; Ding, J.-D.; Zhang, Y.-M.; Lin, Q.; Yao, H.; Wei, T.-B. Chin. J. Org. Chem. 2018, 38, 2741 (in Chinese). (陈进发，刘茜，韩冰冰，丁金东，张有明，林奇，姚虹，魏太保， 有机化学, 2018, 38, 2741.)

[42] Venkatesan, M.; Sathiyanarayanan, K. I. Sens. Actuators B 2018 , $267,373$.

[43] Huang, X.; Wu, S.-S.; Ke, X.-K.; Li, X.-Y.; Du, X.-Z. ACS Appl. Mater. Interfaces 2017, 9, 19638.

[44] Cui, S.-Y.; Du, J.-L.; Wang, T.; Hu, X.-L. Spectrochim. Acta A 2012, 96, 188.

[45] Zhou, Y.-Y.; Yu, H.-P.; Zhang, L.; Xu, H.-W.; Wu, L.; Sun, J.-Y.; Wang, L. Microchim. Acta 2009, 164, 63.

[46] Montes-Navajas P., Corma, A.; Garcia, H. Chem. Phys. Chem. 2008, 9, 713 .

[47] Lu, Q.; Gu, J.-H.; Yu, H.-P.; Liu, C.; Wang, L.; Zhou, Y.-Y. Spectrochim. Acta A 2007, 68, 15

[48] Zhao, G.-C.; Zhu, J.-J.; Chen, H.-Y. Spectrochim. Acta A 1999, 55, 1109.

[49] Yang, K.; Wen, J.; Chao, S.; Liu, J.; Yang, K.; Pei, Y.-X.; Pei, Z.-C. Chem. Commun. 2018, 54, 5911.

[50] Wang, L.; Fan, Y.-Q.; Guan, X.-W.; Qu, W.-J.; Lin, Q.; Yao, H.; Wei, T.-B.; Zhang, Y.-M. Tetrahedron 2018, 74, 4005.

[51] Siangproh, W.; Somboonsuk, T.; Chailapakul, O.; Songsrirote, K. Talanta 2017, 174, 448.

[52] Chuntib, P.; Themsirimongkon, S.; Saipanya, S.; Jakmunee, J. Talanta 2017, 170, 1 .

[53] Zhao, Z.-Z.; Zhang, F.-W.; Zhang, Z.-P. Spectrochim. Acta A 2018, 199, 96

[54] Chuntib, P.; Jakmunee, J. Talanta 2015, 144, 432.

[55] Tu, J.; Xiao, L.-L.; Jiang, Y.-F.; He, Q.-Y.; Sun, S.-G.; Xu, Y.-Q. Sens. Actuators B 2015, 215, 382.

[56] Chen, C.-R.; Men, G.-G.; Bu, W.-H.; Liang, C.-S.; Sun, H.-C.; Jiang, S.-M. Sens. Actuators B 2015, 220, 463.

[57] Hu, S. M.S. Thesis, Hebei University of Science and Technology, Shijiazhuang, 2011 (in Chinese) (胡硕，硕士论文，河北科技大学，石家庄, 2011.)

[58] Grandis, V. D.; Bizzarri, A. R.; Cannistraro, S. J. Mol. Recognit 2007, 20, 215

[59] Hu, X.-Y.; Liu, X.; Zhang, W.-Y.; Qin, S.-Yao, C. H.; Li, Y.; Cao, D.-R.; Peng, L.-M.; Wang, L.-Y. Chem. Mater. 2016, 28, 3778.

[60] Yao, Y. Ph.D. Dissertation, Zhejiang University, Hangzhou, 2015 (in Chinese) (姚勇, 博士论文, 浙江大学, 杭州, 2015.)

[61] Morris, G. M.; Huey, R.; Lindstrom, W.; Sanner, M. F.; Belew, R. K.; Goodsell, D. S.; Olson, A. J. J. Comput. Chem. 2009, 30, 2785.

(Li, L.; Fan, Y.) 